\title{
Sur la décomposition de quelques sulfates acides à la suite d'une déformation mécanique,
}

par M. W. SPRING.

On sait, aujourd'hui, que la compression n'a pas toujours pour effet de diminuer le volume des corps sur lesquels elle s'exerce: quand elle a dépassé une.certaine limite, différente d'une substance à une autre, elle a, au contraire, pour suite, une dilatation de la matière. Ce fait, que j'ai pu observer, il y a déjà longtemps ${ }^{1}$ ), sur quelques substances, a été généralisé récemment par G. Kahlbaum ${ }^{2}$ ).

Dans une note insérée dans le numéro 1 du tome XXIII de ce Recueil, j'ai montré que le bismuth fait exception à la règle générale: il ne se dilate pas sous forte compression, comme le font les autres métaux, mais il se condense de plus en plus. Si l'on fait attention que le bismuth se distingue des autres métaux par ce qu'il ne se dilate pas, comme cenx-ci, quand il entre en fusion, mais qu'il se contracte au contraire, on n'est pas éloigné de penser que le phénomène paradoxal de la dilatation d'un corps pendant sa compression est en relation étroite avec la déformation qu'il éprouve du chef de l'action mécanique auquel il se trouve soumis.

1) Sur l'élasticitéparfaite des corps chimiquement définis (Bull. de l'Acad. Roy. de Belgique, 5e sér., t. VI, 1883).

$\left.{ }^{2}\right)$ Physik. Zeitschrift, p. 32, 1901, et Abhandl. der naturf. Gesellschaft in Basel, t. VI, p. 21.

Rec. d. trav. chim. d. Pays-Bas et de la Belgique. 
J'ai dit, à titre d'hypothèse, dans la note rappelée plus baut, que les déformations mécaniques des corps solides provoquaient, sans doute, un changement de la structure moléculaire en ce sens qu'elles faisaient prendre au corps la formation intérieure répondant à l'état liquide, et cela en deliors d'une élévation notable de la température.

Si cette manière de voir est exacte, on pent s'attendre à trouver la densité des corps diminuée toutes les fois que l'on opère avec une matière se dilatant pendant la fusion, tandis qu'on pourra la trouver plus grande dans le cas contraire. Il m'a paru également, que cette considération était de nature à fournir une explication facile de la plasticité des corps solides, ou bien aussi de leur fragilité. Les corps seraient d'autant plus plastiques, sous les fortes pressions, qu'ils prendraient plus facilement la structure moléculaire à laquelle correspond l'état liquide. Dans les cas où cette condition ne serait pas remplie, le corps se briserail dès l'instant où il ne pourrait plus résister à la déformation que lui imposent les forces mécaniques en jeu.

Cette hypothèse, qui ne s'appuyait alors que sur la particularité présentée par le bismuth comparativement aux autres métaux, demandait un contrôle complémentaine.

Je désire faire connaître, aujourd'hui, quelques résultats d'expériences entreprises $\dot{a}$ cette fin sur certains sels; ils contribueront peut-être à éclairer la question.

Voici la pensée qui a servi de guide dans ces expériences. Si la déformation mécanique d'un corps est de nature a provoquer un changement dans la structure moléculaire de celui-ci, de manière qu'une partie, au moins, du solide accuse les propriétés caractéristiques de l'état liquide, il est probable qu'en agissant sur des corps composés on constatera leur décomposition lorsque ceux-ci résulteront de la combinaison d'un constituant solide et d'un autre liquide à la température ordinaire. La décomposition pourra même s'achever d'autant plus complètement que le constituant liquide s'échappera plus facilement pendant la compression. Les corps composés qui répondent à la condition énoncée sont très nombreux.

Les cristaux renfermant de l'eau de cristallisation sont du nombre; il en est de même des sulfates acides.

Ce sont ceux-là qui ont servi de matériel pour les recherches actuelles. On peut, en effet, regarder le sulfate acide de sodium comme renfermant, potentiellement, le sel neutre et l'acide sulfurique et s'attendre à ce que la déformation mécanique du corps composé réalise l'acide sulfurique à l'état liquide et l'exprime, au moins partiellement, de la masse comprimée.

J'ai fait usage de tous les sulfates acides alcalins, depuis celui de lithium jusqu'à celui de césium, afin de constater comment se modifie la stabilité de ces corps d'un terme à un autre de la série.

Il importe de toncher encore un point essentiel avant de passer à l'exposé des faits observés.

On se rappelle, sans doute, que j'ai déjà produit la décomposition de quelques corps par la seule compression ${ }^{1}$ ); mais alors ces corps avaient été choisis parmi ceux dont le volume moléculaire est plus grand que la somme des volumes des constituants. Par exemple, le volume moléculaire de l'hydrate de sulfure d'arsenic $\left(\mathrm{As}_{2} \mathrm{~S}_{3} \cdot 6 \mathrm{H}_{2} \mathrm{O}\right)$ est

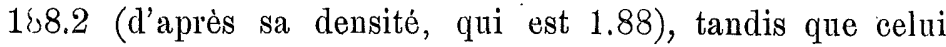
de $\mathrm{As}_{2} \mathrm{~S}_{3}$ étant 71.3 et celui de l'eau $6 \times 18$ ou 108 , leur somme: 179.3 est plus petite que le volume du composé. Aussi est-ce avec facilité que ce sulfure hydraté se décompose sous l'influence d'une compression hydrostatique.

Dans le cas qui nous occupe aujourd'hui, il ne s'agit plus seulement de savoir si la compression décompose les corps tels que l'bydrate de trisulfure d'arsenic, mais de vérifier si, même quand le volume moléculaire du composé

1) Bull. de l'Acad. roy. de Belgique, 3e sér., t. XII, p. 409, 1887, et t. XXX, p. 199, 1895 
est plus petit que la somme des volumes des composants, la compression produit un effet chimique lor'sq ue l'o n permet à la matière desedéformer mécaniquement.

Il ne s'agira donc plus, dans ce qui va suivre, de compression hydrostatique simple, mais plutôt d'un véritable pétrissage ou laminage de la matiëre, en uu mot, du phénomène qui accompagne le flux (ou écoulement solide) de certains corps à l'état solide.

Pendant ce laminage des corps solides, il doit se produire une eertaine élévation de température. On pourrait lui attribuer l'origine des phénomènes de décomposition que l'on va constater, mais il n'en est rien cependant. En effet, la pression a toujours été donnée lentement, par la manouvre d'une vis, de façon à éviter les à-coups qui eussent produit une élévation de température locale; en outre, le compresseur étant tout en métal, devait fonctionner comme un refroidisseur parfait, d'autant que sa masse était infinie, pratiquement, par rapport au poids de matière comprimée, poids qui ne dépassait jamais 3 grammes. Je ne suis pas parvenu, au surplus, à enflammer de la poudre à tirer en la soumettant aux mêmes essais. On peut donc être certain que la température ne monte pas même au point de fusion du soufre.

Nous examinerons d'abord les volumes moléculaires des sulfates dont nous nous sommes servis.

Nous pourrons combler, de cette façon, quelques lacunes dans la connaissance de la densité de ces corps et rectifier même une erreur, assez forte, qui se trouve reproduite dans tous les ouvrages que nous avons pu consulter, sur la densité du sulfate acide de sodium.

Les sulfates acides ont été préparés eu fondant ensemble, à la plus basse température possible, les quantités équivalentes de sulfates neutres, purs, et d'acide sulfurique. Il s'agit donc, exclusivement, pour l'instant, de sulfates acides ne renfermant pas d'eau de cristallisation.
La densité a été prise par la méthode hydrostatique, au sein de benzène pur et rapportée à l' e a u par le calcul.

Pour rendre la comparaison plus aisée, on a réuni les résultats des observations, tant anciennés que nouvelles, dans le tableau suivant, où figurent, d'ailleurs aussi, les données relatives aux volumes moléculaires des sulfates acides et aux volumes de leurs constituants.

\begin{tabular}{l|c|c|c|l}
\hline $\begin{array}{c}\text { Sub- } \\
\text { stances. }\end{array}$ & $\begin{array}{c}\text { Densités } \\
\text { admises. }\end{array}$ & $\begin{array}{c}\text { Observations } \\
\text { nouvelles. }\end{array}$ & $\begin{array}{c}\text { Poids } \\
\text { moléculaires. }\end{array}$ & Volumes moléculaires de \\
\hline $\mathrm{Li}_{2} \mathrm{SO}_{4} \cdot$ & $\left.2.210^{1}\right)$ & 2.228 & 110 & $2 \mathrm{LiHSO}_{4}=98.1$ \\
$\mathrm{LiHSO}_{4} \cdot$ & - & 2.123 & 104 & $\mathrm{Li}_{2} \mathrm{SO}_{4}+\mathrm{H}_{2} \mathrm{SO}_{4}=10.23$ \\
$\mathrm{Na}_{2} \mathrm{SO}_{4}$ & $\left.2.655^{1}\right)$ & - & 142 & $2 \mathrm{NaHSO}_{4}=98.6$ \\
$\mathrm{NaHSO}_{4} \cdot$ & $\left.2.742^{2}\right)$ & 2.435 à $13^{\circ}$ & 120 & $\mathrm{Na}_{2} \mathrm{SO}_{4}+\mathrm{H}_{2} \mathrm{SO}_{4}=106.4$ \\
$\mathrm{~K}_{2} \mathrm{SO}_{4}$ & $\left.2.670^{1}\right)$ & - & 174 & $2 \mathrm{KHSO}_{4}=118$ \\
$\mathrm{KHSO}_{4} \cdot$ & 2.355 & 2.302 à $13^{\circ}$ & 136 & $\mathrm{~K}_{2} \mathrm{SO}_{4}+\mathrm{H}_{2} \mathrm{SO}_{4}=118.1$ \\
$\mathrm{Rb}_{2} \mathrm{SO}_{4}$ & $\left.3.611^{2}\right)$ & 3.596 à $16^{\circ}$ & 266 & $2 \mathrm{RbHSO}_{4}=125 . \mathrm{s}^{\circ}$ \\
$\mathrm{RbHSO}_{4} \cdot$ & - & 2.892 à $16^{\circ}$ & 182 & $\mathrm{Rb}_{2} \mathrm{SO}_{4}+\mathrm{H}_{2} \mathrm{SO}_{4}=126.9$ \\
$\mathrm{Cs}_{2} \mathrm{SO}_{4} \cdot$ & $\left.4.243^{2}\right)$ & 4.250 à $16^{\circ}$ & 360 & $\mathrm{CsHSO}_{4}=136.6$ \\
$\mathrm{CsHSO}_{4} \cdot$ & - & 3.352 à $16^{\circ}$ & 229 & $\mathrm{Cs}_{2} \mathrm{SO}_{4}+\mathrm{H}_{2} \mathrm{SO}_{4}=137.7$ \\
& & &
\end{tabular}

N.B. Le volıme moléculaire de $\mathrm{H}_{2} \mathrm{SO}_{4}$ a été calculé en prenant 1.842 pour la densité de cet acide; on a alors: $98: 1.842=53.2$.

Il résulte de la comparaison des nombres figurant dans la dernière colonne de ce tableau que le volume moléculaire des sulfates acides alcalins est toujours plus petit que la somme des volumes de leur's constituants. D'après cela, une simple compression hydrostatique ne peut provoquer leur décomposition, et si celle-ci se produit néanmoins à la suite de déformations mécaniques, c'est que les déformations sont

1) Tabellen von Landolt und Börnstein.

2) Damarer, Suppl. pp. 460 à 468. 
en état d'atteindre la structure moléculaire. C'est ce que nous verrons dans le paragraphe suivant.

Remarquons encore que la densité généralement admise pour $\mathrm{NaHSO}_{4}$, d'après les travaux de Playfair et Joule, est trop grande de plus de $10 \%$. En l'acceptant, on trouve pour le volume moléculaire de $\mathrm{NaHSO}_{4}$, le nombre 87.6, qui est tout à fait en dehors de la norme fournie par l'ensemble des autres sels.

Nous arrivons à présent aux résultats obtenus à la suite des déformations mécaniques.

Pour réaliser la déformation désirée, on a comprimé les sulfates acides dans un cylindre en acier dont le fond était percé d'un trou. La matière fluait lentement par cet orifice sitôt que la pression était assez grande. En outre, le piston compresseur a été façonné de manière à ne pas fermer. absolument le cylindre; il descendait donc en quelque sorte dans la matière et la laminait fortement contre les parois du cylindre.

\section{1. - Sulfate acide de lithium.}

Le sel préparé par fusion, absolument sec, donne naissance, par suite de sa déformation, à de l'acide sulfurique libr'e qui s'écoule, d'une manière visible, mêlé de sel solide, par l'orifice du compresseur. On a séparé la partie du sel qui avait flué de celle qui était restée en masse sous le piston et on a dosé le lithium dans chacune d'elles, par calcination jusqu'à retour de la matière à l'état neutre $\mathrm{Li}_{2} \mathrm{SO}_{4}$.

On a trouvé:

\begin{tabular}{c|c|c|c|c}
\hline & $\begin{array}{c}\text { Partie } \\
\text { qui a flué. }\end{array}$ & $\begin{array}{c}\text { Partie } \\
\text { qui n’a pas flué }\end{array}$ & $\begin{array}{c}{\mathrm{Le} \mathrm{Li}_{2} \mathrm{SO}_{4}}_{\text {renferme }} \\
\text { renferme }\end{array}$ & $\begin{array}{c}\mathrm{Le} \mathrm{Li} \mathrm{HSO}_{4} \\
\text { renferme }\end{array}$ \\
\hline $\mathrm{Li} \%$ & 5.62 & 7.39 & 12.72 & 6.73
\end{tabular}

Ce résultat est frappant: le sel $\mathrm{LiHSO}_{4}$ s'est partagé sous l'action de la déformation en une partie plus acide, renfermant moins de lithium que la matière première, qui s'est écoulée en partie et une autre, moins acide, qui est restée dans le cylindre. Il est facile de calculer, à l'aide des nombres précédents, que la partie qui a flué renfermait environ $17 \%$ d'acide sulfurique libre; elle peut se traduire, approximativement, par la formule $9 \mathrm{LiHSO}_{4} \cdot 2 \mathrm{H}_{2} \mathrm{SO}_{4}$, qui montre mieux la proportion d'acide devenu libre.

A la suite de ce résultat, on a préparé du sulfate acide de lithium hydraté $\mathrm{LiHSO}_{4} \cdot \mathrm{H}_{2} \mathrm{O}$ en dissolvant le sel sec dans de l'acide sulfurique hydralé chaud et en laissant cristalliser la masse.

Les cristaux desséchés dans un exsiccateur ont donné $5.85 \%$ de lithium à l'analyse, tandis que $\mathrm{LiHSO}_{4} \cdot \mathrm{H}_{2} \mathrm{O}$ en renforme, théoriquement, 5.73 .

Ce sel donne abondamment un liquide acide pendant la compression. Le résidu solide resté dans le cylindre du compresseur a donné $10.8 \%$ de lithium à l'analyse. A l'aide de ce renseignement, on calcule que ce résidu solide devait se composer de

$$
\begin{aligned}
& 87.43 \% \text { de } \mathrm{Li}_{2} \mathrm{SO}_{4} \text {, et } \\
& 12.57 \% \text { de } \mathrm{LiHSO}_{4}, \mathrm{H}_{2} \mathrm{O} .
\end{aligned}
$$

Ce sel hydraté est donc beaucoup plus fragile que le précédent.

Il est à supposer que si la compression pouvait s'exercer de manière que toutes les parties de la matière essayée participent à une déformation mécanique égale et suffisante, tout en permettant un écoulement complet de l'eau et de l'acide sulfurique, la décomposition s'achèverait tout à fait. 


\section{2. - Sulfate acide de sodium}

Le sel acide sec $\mathrm{NaHSO}_{4}$, obtenu par fusion, s'est montré d'une rigidité telle qu'il n'a pas été possible de le faire fluer en exerçant même la pression la plus forte compatible avec la solidité de l'appareil. En ouvrant le cylindre du compresseur, on a trouvé un bloc de sel compact accusant un commencement de fl $\mathrm{ux}$ autour du piston, mais sans résultat décisif au regard de la décomposition.

On a procédé alors à la préparation de sels hydratés.

En soumettant à la cristallisation une solution du sel précédent dans de l'acide sulfurique additionné d'une proportion égale d'eau, on a obtenu, d'abord, de gros prismes. Soit A ce sel: Après, il s'est formé de fines aiguilles d'un autre sel: désignons le par $B$.

L'analyse de ces sels a donné :

$$
\begin{array}{l|c|c}
\hline \hline & \text { Sel A. } & \text { Sel B. } \\
\hline \text { Na . . . } & 16.26 \% & 1235 \% \\
\text { S. . . . } & 23.63 \% & 20.03 \%
\end{array}
$$

$D^{\prime}$ où l'on déduit les formules:

$\mathrm{A}=\mathrm{NaHSO}_{4}, \mathrm{H}_{2} \mathrm{O}$ (qui contient $\mathrm{Na}=16.66 \%$ et $\mathrm{S}=23.19 \%$ )

$\mathrm{B}=6 \mathrm{NaHSO}_{4}, \mathrm{H}_{2} \mathrm{SO}_{4}, 16 \mathrm{H}_{2} \mathrm{O}$ (qui contient $\mathrm{Na}=12.47 \%$ et $\mathrm{S}=20.25 \%$ ).

La densité du sel A a été trouvée égale à 2.103 à $13^{05}$; on en déduit pour $\mathrm{NaHSO}_{4} \cdot \mathrm{H}_{2} \mathrm{O}$, le volume moléculaire 65.6 ; or le volume moléculaire de $\mathrm{NaHSO}_{4}$ étant 49.3 et celui de $\mathrm{H}_{2} \mathrm{O}, 18$, on a, pour la somme des deux, 67.3, donc la compression seule ne doit pas provoquer la décomposition de ce corps.

En soumettant ce corps aux déformations mécaniques, on constate qu'il n'y a pas de décomposition certaine, la température étant de $10^{\circ}$. La partie non fluée du sel contenait $\mathrm{Na}=16.67 \%$ contre 16.26 ; différence qui ne permet pas de conclure.
Mais en opérant à une température plus élevée, à $30^{\circ}$, la décomposition commence; le sel flué contient alors $17.25 \%$ de $\mathrm{Na}$, et si l'on opère à $40^{\circ}$, on y trouve $18.28 \%$ de $\mathrm{Na}$.

Le sel perd principalcment son ea u d'hydiratation. Si l'on admet, par hypothèse, que celle-ci n'entraîne pas d'acide, on peut calculer que $64.5 \%$ de la quantité d'eau possible ont été expulsés lors de la compression à $40^{\circ}$.

Quant au sel B, il est très fragile. Comprimé à la température du laboratoire $\left(10^{\circ}\right.$ ce jour-là), il donne beaucoup de liquide acide. La masse restée dans le compresseur a fourni $\mathrm{Na}=18.81 \%$ à l'analyse. Comme le sel $\mathrm{NaHSO}_{4}$ contient $19.17 \%$ de $\mathrm{Na}$, on peut en conclure que le sel $\mathrm{B}$ devient presque totalement du sulfate acide $\mathrm{NaHSO}_{4}$ par la déformation. La décomposition s'arrête alors, ce qui est conforme au fait mentionné plus haut.

A titre de contrôle des résultats précédents, on a préparé un sulfate acide de sodium en saturant un mélange d'acide sulfurique et d'eau, en proportions à peu près égales, de sulfate de sodium cristallisé $\left(\mathrm{Na}_{2} \mathrm{SO}_{4} \cdot 10 \mathrm{H}_{2} \mathrm{O}\right)$ à la température de $50^{\circ}$ environ et en laissant refroidir. Il s'est formé des cristaux dont la composition était: $5\left(\mathrm{NaHSO}_{4}\right)+$ $\mathrm{H}_{2} \mathrm{SO}_{4}+7 \mathrm{H}_{2} \mathrm{O}$; en effet, on a:

\begin{tabular}{l|c|c}
\hline & Analyse & Calcul. \\
\hline $\mathrm{Na} \ldots .$. & 13.95 & 1395 \\
$\mathrm{~S} \ldots .$. & 22.84 & 23.29
\end{tabular}

Ce sel perd totalement son eau quand on l'abandonne dans le vide sec, après un mois environ. Le sel restant répoudait à la formule $5\left(\mathrm{NaHSO}_{4}+\mathrm{H}_{2} \mathrm{SO}_{4}\right)$; en effet, on trouve:

\begin{tabular}{l|c|c}
\hline & Analyse. & Calcul. \\
\hline $\mathrm{Na} \ldots .$. & 16.83 & 16.47 \\
$\mathrm{~S} \ldots . .$. & 27.52 & 27.50
\end{tabular}


Il paraît abandonner plus facilement 4 de ses molécules d'eau, car on obtient la composition suivante après quelques jours de dessiccation dans un exsiccateur, sous pression atmosphérique, $5 \mathrm{NaHSO}_{4} \cdot \mathrm{H}_{2} \mathrm{SO}_{4} \cdot 3 \mathrm{H}_{2} \mathrm{O}$, car

\begin{tabular}{c|c|c}
\hline & Analyse. & Calcul. \\
\hline Na . . . & 15.37 & 15.29 \\
S . . . . & 25.62 & 25.63
\end{tabular}

(On trouve dans Dammer, t. II, 2, p. 161, que Brindley a déjà obtenu le sel $\mathrm{NaHSO}_{4} \cdot \mathrm{H}_{2} \mathrm{SO}_{4}$. Comme il l'a formé en chauffant $\mathrm{NaHSO}_{4}+\mathrm{H}_{2} \mathrm{SO}_{4}$ entre $200^{\circ}$ et $300^{\circ}$, il ne pouvait obtenir, naturellement, les produits hydratés dont il est fait mention ici.)

Si l'on soumet le sel à $7 \mathrm{H}_{2} \mathrm{O}$ aux déformations mécaniques à la température de $40^{\circ}$, il y a dégagement abondant de liquide acide; la partie qui n'a pas flué était sèche en apparence, elle a donné, à l'analyse:

$$
\begin{aligned}
\mathrm{Na} & =1.9 .15 \% \\
\mathrm{~S} & =26.56 \%
\end{aligned}
$$

c'est-à-dire qu'elle représentait le $\mathrm{NaHSO}_{4}$ pur; celui-èi contient, en effet:

$$
\begin{aligned}
\mathrm{Na} & =19.16 \% \\
\mathrm{~S} & =26.66 \%
\end{aligned}
$$

Ainsi donc, le laminage du sel a eu pour effet de dégager complètemient l'acide sulfurique et l'eau qui formaient corps avec le $\mathrm{NaHSO}_{4}$; mais ici aussi, la décomposition n'a pas été plus loin.

Eufin, si l'on opère à l'aide du sel à $3 \mathrm{H}_{2} \mathrm{O}$, à la température du laboratoire $\left(16^{\circ}\right)$, il y a dégagement d'eau. La partie restée dans le compresseur renferme:

$$
\begin{aligned}
\mathrm{Na} & =16.66 \% \\
\mathrm{~S} & =26.84 \%,
\end{aligned}
$$

c'est-à-dire qu'on doit la regarder comme répondant à la formule $5 \mathrm{NaHSO}_{4} \cdot \mathrm{H}_{2} \mathrm{SO}_{4}$ (voir plus haut pour la composition). Il en résulte qu'à basse température, l'ea u cèd e la première a ux efforts mécaniques. C'est d'ailleurs dans l'ordre des affinités.

Pour vérifier l'effet produit par une température plus élevée sur ce sel à $3 \mathrm{H}_{2} \mathrm{O}$, j'ai chauffé le compresseur à $80^{\circ}$. Pendant le travail mécanique, il s'est écoulé beaucoup de liquide acide. Le résidu demeuré dans l'appareil renfermait:

$$
\begin{aligned}
\mathrm{Na} & =19.20 \\
\mathrm{~S} & =24.40 .
\end{aligned}
$$

Ces nombres se rapprochent beaucoup de ceux que demande la formule $\mathrm{NaHSO}_{4}$, savoir $\mathrm{Na}=19.16$ et $\mathrm{S}=26.66$, mais le $d$ éfau $t$ de $S$ engage à calculer le rapport atomique $\frac{S}{\mathrm{Na}} q u i$ est naturellement l'unité dans $\mathrm{NaHSO}_{4}$. On trouve:

$$
\begin{aligned}
& 19.20: 23=0.835 \\
& 24.40: 32=0.762
\end{aligned} \text { d'où }: \frac{0.762}{0.835}=0.912=\frac{\mathrm{S}}{\mathrm{Na}}
$$

le rapport étant plus petit que l'unité d'une grandeur dépassant de beaucoup les erreurs possibles dans un dosage si simple que celui de $\mathrm{Na}$ et $\mathrm{du} \mathrm{S}$, on en doit conclure qu'une certaine proportion de $\mathrm{NaHSO}_{4}$ a dû passer à l'état de $\mathrm{Na}_{2} \mathrm{SO}_{4}$, très approximativement en quantité telle que le produit final devrait avoir une composition donnant $11 \mathrm{Na}$ pour $10 \mathrm{~S}, \operatorname{car} \frac{10}{11}=0,909$; ce qui se traduirait par la formule: $\mathrm{Na}_{2} \mathrm{SO}_{4}+9 \mathrm{NaHSO}_{4}$. En un mot, à une température plus élevée, il n'y a pas que le $\mathrm{H}_{2} \mathrm{SO}_{4}$ combiné à $\mathrm{NaHSO}_{4}$ qui cède, mais le $\mathrm{NaHSO}_{4}$ lui-même commence à se disloquer sous l'influence des efforts mécaniques. Il est probable qu'en opérant à une température plus élevée encore, les résultats seraient aussi plus complets et que l'on arriverait à dégager tout l'acide sulfurique contenu, en puissance, dans le sulfate acide de sodium. 
3. - Sulfates acides de potassium, de rubidium, de césium.

La stabilité, ou la solidité, de ces sulfates est telle qu'aucun d'eux ne se décompose sous les efforts mécaniques, même quand on opère à une température peu différente de $100^{\circ}$. Ce résultat négatif est à rapprocher du fait que ces sulfates acides se décompusent bien plus difficilement que les précédents sous l'influence de la chaleur. Il paraît donc exister une certaine connexion entre la stabilité chimique et la stabilité mécanique.

On a préparé aussi dı sulfate de potassium renfermant une proportion plus grande d'acide libre, en dissolvant le sel $\mathrm{KHSO}_{4}$ dans de l'acide sulfurique chaud, dilué d'eau et laissant ensuite cristalliser par refroidissement.

Il s'est for'ıé des aiguilles cristallines dont la composition peut être exprimée par la formule $2 \mathrm{KHSO}_{4} 3 \mathrm{H}_{2} \mathrm{SO}_{4} 3 \frac{1}{2} \mathrm{H}_{2} \mathrm{O}$, car on a:

\begin{tabular}{l|c|c}
\hline & Analyse. & Calcul. \\
\hline K . . . . & 12.30 & 1230 \\
S. . . . & 25.36 & 25.43
\end{tabular}

Ce sel n'a pas résisté aux efforts mécaniques, non plus que les sels de sodium analogues dont il a été question. A la température ordinaire, déjà, il s'est dégagé beancoup de liquide. Le corps solide resté dans le compresseur avait, approximativement, la composition $\mathrm{KHSO}_{4}, \mathrm{H}_{2} \mathrm{SO}_{4}, \mathrm{H}_{2} \mathrm{O}$, car on a:

\begin{tabular}{c|c|c}
\hline & T'rouvé. & Calculé. \\
\hline K. . . . . & 14.77 & 15.14 \\
S. . . . & 25.12 & 25.39
\end{tabular}

On voit done qu'une quantité notable d'acide sulfurique est devenue libre pendant le travail mécaniqne.

On n'a pas préparé de sels plus acides avec les sulfates de rubidium et de césium.
En vue de vérifier plus complètement encore la part qui revient à la déformation mécanique de la matière dans les phénomènes précédents, on a soumis à la compreșsion seule d'abord, et ensuite à la déformation, des mélanges intimes de sulfate acide de sodium sec avec divers oxydes basiques: $\mathrm{PbO}, \mathrm{CuO}, \mathrm{HgO}, \mathrm{Ag}_{2} \mathrm{O}$ également secs.

Pour réaliser la compression simple, sans déformation mécanique notable, on a enserré la poudre des mélanges à examiner, dans le cylindre du compresseur, entre deux petits pistons de cuivre bien ajustés. Ces pistons, s'écrasant encore sous l'action de la pression, fermajent le cylindre d'une manière complète et empêchaient un laminage de la matière.

Dans ces conditions, les poudres employées sont devewues des cylindres compacts, très solides, mais sans apparence de réaction chimique. La grande solidité de ces cylindres fait cependant présumer qu'une réaction peut avoir eu lieu à la surface des grains sans avoir gagné la profondeur. La preuve en est que la couleur du mélange n'a pas changé.

Par exemple, le mélange $2 \mathrm{NaBSO}_{4}+\mathrm{CuO}$ est noir et il est resté noir après la compression, tandis que les produits de la réaction: $\mathrm{Na}_{2} \mathrm{SO}_{4}+\mathrm{CuSO}_{4}+\mathrm{H}_{2} \mathrm{O}$, sont blancs ou peu s'en faut.

Tout autre est le résultat quand une déformation mécanique accompagne la compression. Alors la couleur du mélange change rapidement et la réaction s'achève au point que la neutralisation du sel acide a lieu totalement.

On peut constater ces faits plus simplement encore. Il suffit de broyer énergiquement le sulfate acide de sodium et l'oxyde de cuivre dans un mortier en agate pour voir la couleur foncée de la poudse primitive devenir plus claire et la réaction s'achever lentement au point que toute la matière peut être dissoute dans l'eau sans qu'il demeure de résidu de CuO. L'eau produite pendant la réaction paraît se combiner aux sels à mesure de sa formation, car le produit final est sec et blanc; il ne perd que très peu de son poids quand on l'abandonne dans un exsiccateur. 
Résumé et conclusions.

Il résulte de ce qui précède que les sulfates acides des métaux alcalins ne résistent pas tous, chimiquement, à une déformation mécanique. Ceıx qui dérivent du lithium et du sodium, et surtont les sulfates renfermant une plus grande proportion moléculaire d'acide sulfurique et d'eau d'hydratation, se décomposent alors de manière à abandonner une grande partie de leur ean et une quantité assez notable d'acide sulfurique pour qu'il y ait même production d'une certaine quantité de sulfate neutre. Les sulfates dérivant des métaux alcalins de poids atomique plus élevé résistent à la décomposition, du moins dans les conditions de température et de pression qui ont été réalisées.

La décomposition dont il s'agit ici n'est pas la conséquence immédiate de la compression, c'est-à-dire de la simple diminution de volume provoquée par la pression; mais elle est l'effet du laminage ou du pétrissage de la matière.

Il résulte donc de là que la déformation mécanique peut produire la décomposition de certains corps; elle semble agir alors à la manière d'une élévation de la température et peut aussi remplacer celle-ci. Un corps solide résiste d'autant mieux à la décomposition par déformation, dans un groupe donné de substances, qu'il est plus réfractaire à l'action de la chaleur. Il est à remarquer aussi que les combinaisons dites moléculaires, paraissent résister moins bien aux déformations mécaniques que les combinaisons a tomiques proprement dites. Si cette observation se vérifie par la suite, il y aura sans doute lien de faire une distinction mécanique réelle, de ces deux ordres de combinaisons.

Cette décomposition mécanique trouve une explication aisée si l'on admet ${ }^{1}$ ) que, pendant sa déformation mécanique forcée, le corps solide peut prendre, l'état moléculaire dans lequel le frottement intérieur est plus

1) Voir ma note Sur la diminution de densitédescorps par compression (Loc. cit.). faible, en d'autres termes, qu'il passe alors à un état de pseudo-fusion ou de pseudo-liquéfaction, en tout ou en partie, de même que, dans d'autres circonstances, la compression favorise le passage d'un état allotropique à un autre. Alors il est concevable que les corps composés susceptibles de donner naissance, par changement de structure moléculaire, à des corps liquides véritablement, à la température de l'expérience, ne passent pas seulement à un état de pseudo-liquéfaction, mais qu'ils se décomposent dans leurs constituants, au moins pendant toute la durée de l'acte mécanique. Si, par les dispositions prises, on permet à la partie liquide de se dégager, le phénomène, pourra être constaté; dans le cas contraire, il échappera généralement à l'observation.

Cette décomposition des corps solides par voie de laminage ou d'écoulement, à la température ordinaire, peut nous éclairer sur certains phénomènes de métamorphisme fréquemment observés en géologie, pour l'explication desquels on a été obligé de recourir à l'hypothèse, souvent peu probable, d'une élévation locale de la température. Il arrive parfois, on le sait, que la composition des roches n'est pas la même dans les parties qui portent les marques d'un flux ou d'un écoulement. On trouvelà des minéraux microscopiques dont l'origine n'est pas claire. Il est permis de se demander si leur formation ne rentre pas dans l'ordre des faits qui viennent d'être touchés, et si l'écorce terrestre n'a pas été le siège d'un vaste travail mécanico-cbimique qui a éliminé, ou transformé, les corps qu'elle renfermait à l'origine, de telle sorte qu'il ne subsiste plus aujourd'hui que ceux dont la stabilité mécanique a pu triompher des efforts de destruction auxquels ils se trouvaient soumis?

Je me propose de compléter ces recherches.

Mars 1904.

Liége, Institut de chimie générale. 